\title{
Sema3E-PlexinD1 signaling selectively suppresses disoriented angiogenesis in ischemic retinopathy in mice
}

\author{
Yoko Fukushima, ${ }^{1,2,3}$ Mitsuhiro Okada, ${ }^{2}$ Hiroshi Kataoka, ${ }^{2}$ Masanori Hirashima, ${ }^{1}$ Yutaka Yoshida, ${ }^{4}$ \\ Fanny Mann, ${ }^{5}$ Fumi Gomi, ${ }^{3}$ Kohji Nishida, ${ }^{3}$ Shin-Ichi Nishikawa, ${ }^{2}$ and Akiyoshi Uemura1,2,6 \\ 1Division of Vascular Biology, Department of Physiology and Cell Biology, Kobe University Graduate School of Medicine, Kobe, Japan. \\ 2Laboratory for Stem Cell Biology, RIKEN Center for Developmental Biology (CDB), Kobe, Japan. 3Department of Ophthalmology, \\ Osaka University Medical School, Osaka, Japan. ${ }^{4}$ Division of Developmental Biology, Cincinnati Children's Hospital Medical Center, Cincinnati, Ohio, USA. \\ ${ }^{5}$ Developmental Biology Institute of Marseille Luminy, CNRS UMR 6216, University of the Mediterranean, Marseille, France. \\ ${ }^{6}$ Division of Ophthalmology, Department of Surgery, Kobe University Graduate School of Medicine, Kobe, Japan.
}

\begin{abstract}
During development, the retinal vasculature grows toward hypoxic areas in an organized fashion. By contrast, in ischemic retinopathies, new blood vessels grow out of the retinal surfaces without ameliorating retinal hypoxia. Restoration of proper angiogenic directionality would be of great benefit to reoxygenize the ischemic retina and resolve disease pathogenesis. Here, we show that binding of the semaphorin $3 \mathrm{E}$ (Sema3E) ligand to the transmembrane PlexinD1 receptor initiates a signaling pathway that normalizes angiogenic directionality in both developing retinas and ischemic retinopathy. In developing mouse retinas, inhibition of VEGF signaling resulted in downregulation of endothelial PlexinD1 expression, suggesting that astrocyte-derived VEGF normally promotes PlexinD1 expression in growing blood vessels. Neuronderived Sema3E signaled to PlexinD1 and activated the small GTPase RhoJ in ECs, thereby counteracting VEGF-induced filopodia projections and defining the retinal vascular pathfinding. In a mouse model of ischemic retinopathy, enhanced expression of PlexinD1 and RhoJ in extraretinal vessels prevented VEGFinduced disoriented projections of the endothelial filopodia. Remarkably, intravitreal administration of Sema3E protein selectively suppressed extraretinal vascular outgrowth without affecting the desired regeneration of the retinal vasculature. Our study suggests a new paradigm for vascular regeneration therapy that guides angiogenesis precisely toward the ischemic retina.
\end{abstract}

\section{Introduction}

In ischemic retinopathies, including proliferative diabetic retinopathy (PDR) and retinopathy of prematurity (ROP), hypoxia that follows retinal capillary obliteration drives deregulated growth of new blood vessels protruding out of the retinal surfaces, without ameliorating retinal ischemia (1-3). Sustained hypoxia further exacerbates extraretinal vascular outgrowth, which can cause vision-impairing hemorrhage and retinal detachment. Therefore, it has been widely assumed that blood vessel formation per se should be prevented to treat these common blinding diseases (4). A series of anti-VEGF drugs are now being clinically evaluated for their antiangiogenic potency in treating $\operatorname{PDR}$ and $\operatorname{ROP}(5,6)$. However, to fundamentally resolve disease pathogenesis, the promotion of properly guided angiogenesis to reoxygenize the ischemic retina would be of far greater benefit. In this context, it is crucial to identify the molecular targets that can selectively prevent disoriented angiogenesis and thus facilitate retinal vascular regeneration.

For the elaboration of therapies to guide new blood vessels toward the hypoxic retina, the molecular mechanisms underlying intraretinal angiogenesis during developmental periods provide invaluable information. In developing mouse retinas, astrocytes trigger postnatal onset of radial vascular extension from the optic disc by secreting VEGF (also known as VEGF-A) and depositing ECM scaffolds to which the migrating ECs adhere $(7,8)$. Subse-

Conflict of interest: The authors have declared that no conflict of interest exists. Citation for this article: J Clin Invest. 2011;121(5):1974-1985. doi:10.1172/JCI44900. quently, a monolayered vascular network is formed over the superficial retinal area within the first postnatal week. In this process, the concentration gradients of VEGF proteins along the preexisting astrocyte network promote the projection of endothelial filopodia from the tips of sprouting vessels, thereby determining angiogenic orientation (9). On the other hand, diffusible VEGF proteins may induce disoriented filopodia projections $(9,10)$, which should be pruned to restrict retinal vascular pathfinding.

To date, several signaling molecules that guide axonal pathfinding have also been shown to participate in the control of angiogenic orientation (11). In particular, direct binding of the soluble semaphorin 3E (Sema3E) ligand to the transmembrane PlexinD1 receptor, which can act as either an attractive or repulsive cue on neurite extension $(12,13)$, mediates filopodium retraction in growing blood vessels (14) by inactivating R-Ras and activating Arf6 small GTPases $(15,16)$, which leads to the disassembly of integrinmediated adhesive structures. However, the question remains: how does Sema3E-PlexinD1 signaling cooperate with VEGF signaling in defining vascular pathfinding in developing retinas? Moreover, little is known about what signaling defects are responsible for the disoriented vascular outgrowth in ischemic retinopathies.

Here we show that ECs of the developing retinal vessels express PlexinD1 in response to VEGF signaling. Neuron-derived Sema3E signals via PlexinD1 and counteracts VEGF-induced projection of endothelial filopodia by activating the small GTPase RhoJ. In a mouse model of ischemic retinopathy, prominent expression of endothelial PlexinD1 and RhoJ was limited to the abnormal 
A

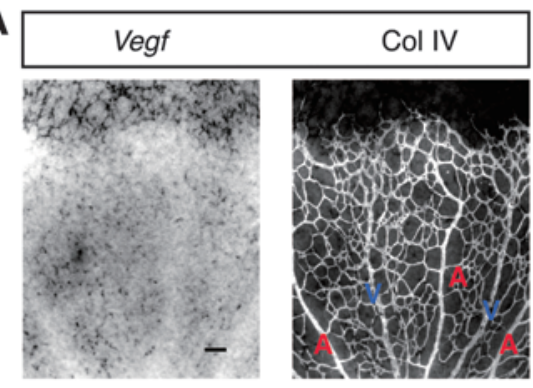

C

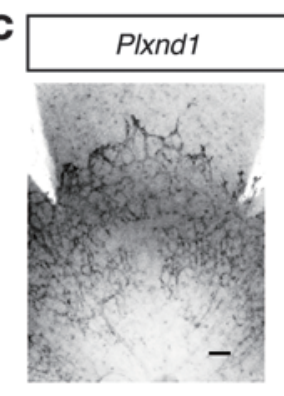

E

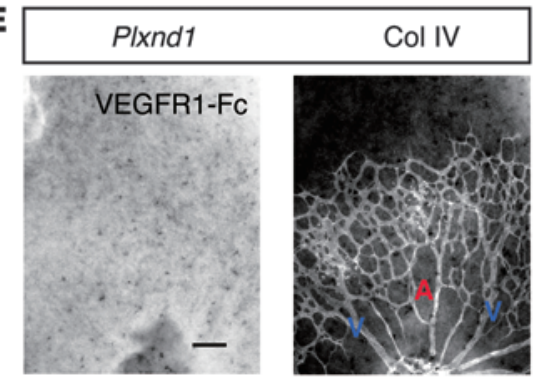

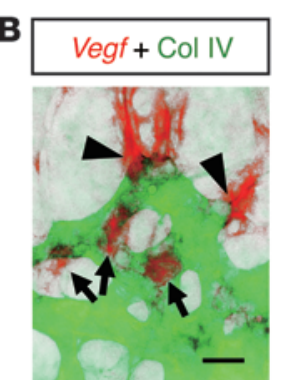
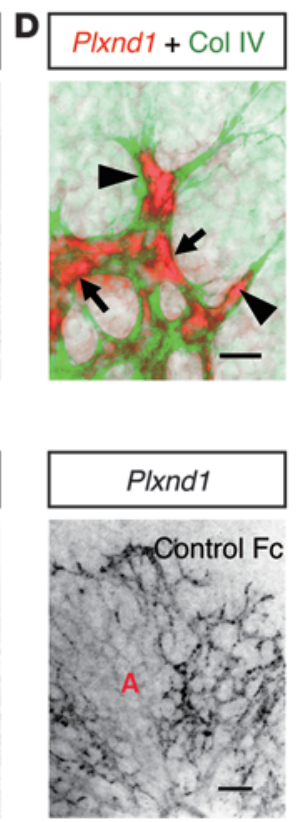

extraretinal vessels. By targeting PlexinD1, intravitreal injection of Sema3E protein selectively suppressed disoriented outgrowth of extraretinal vessels, leading to the subsequent regeneration of normal vasculature in ischemic retinas.

\section{Results}

ECs express PlexinD1 in response to VEGF signaling in the developing retinal vasculature. To explore a therapeutic approach that restores proper angiogenic orientation in ischemic retinopathies, we attempted to exploit the molecular mechanisms underlying intraretinal angiogenesis during development, with special attention to those regulating endothelial filopodia projections. Starting from this framework, we initiated our study by detecting the spatial association of PlexinD1-expressing ECs with VEGF-expressing astrocytes in $\mathrm{P} 4$ mouse retinas, in which blood vessels were growing within the superficial area. As previously reported $(9,10,17)$, the Vegf gene was strongly expressed in retinal astrocytes ahead of the advancing vascular fronts, while moderate Vegf expression was maintained around the preformed capillaries and veins, but not around arteries (Figure 1, A and B). This expression pattern of the Vegf gene can be ascribed to different oxygen concentrations depending on the type of vessel (18). By contrast, the Plxnd1 gene is strongly expressed in ECs located at the tips and in the stalks of sprouting vessels (Figure 1, C and D). In addition, Plxnd1 is moderately expressed in preformed capillaries and veins, but not

\section{Figure 1}

ECs express PlexinD1 in response to VEGF signaling in the developing retinal vasculature. (A) Whole-mount ISH for Vegf in combination with IHC for type IV collagen (Col IV) in the P4 retina. Vegf is expressed in astrocytes ahead of the advancing vessels and around the preformed capillaries and veins, but not around arteries. (B) High-magnification image of the advancing vascular front. Vegf-expressing astrocytes (red) reside adjacent to the tip (arrowhead) and stalk (arrows) ECs (green). (C) ISH for PIxnd1 in combination with IHC for Col IV in the P4 retina. Plxnd1 is expressed in ECs of the developing retinal vasculature, except in arteries. (D) High-magnification image of the advancing vascular front. Plxnd1 (red) is expressed in the tip (arrowheads) and stalk (arrows) ECs (green). (E) ISH for PIxnd1 and IHC for Col IV in P4 retinas 8 hours after intravitreal protein injection. Endothelial Plxnd1 expression is downregulated by VEGFR1-Fc, whereas neuronal PIxnd1 expression is maintained in a dot-like pattern. Note the faint Plxnd1 expression in arteries in the control retina. A, artery; V, vein. Scale bars: $100 \mu \mathrm{m}$ (A, C, and E); $20 \mu \mathrm{m}$ (B and D).

in arteries (Figure 1C). Thus, it seems likely that PlexinD1-expressing ECs reside adjacent to VEGF-expressing astrocytes. To determine whether the expression level of the endothelial Plxnd1 gene fluctuates by manipulating VEGF signaling, we examined Plxnd1 expression after intravitreal injection of a soluble VEGFR1 protein (VEGFR1-Fc), which functions as a high-affinity decoy receptor for VEGF (19). After VEGFR1-Fc injection, endothelial Plxnd1 expression was downregulated at around 7 hours (Supplemental Figure 1; supplemental material available online with this article; doi:10.1172/JCI44900DS1), and became undetectable at 8 hours throughout the developing retinal vasculature (Figure 1E). These results indicate that ECs express PlexinD1 in response to VEGF signaling in growing retinal vessels.

PlexinD1 signaling counteracts VEGF-induced projections of endothelial filopodia. To determine the role of PlexinD1 signaling in retinal vascular development, we pharmacologically neutralized its endogenous ligands by intravitreal injection of its soluble protein, PlexinD1-Fc (20). Five hours after PlexinD1-Fc injection at P5, we observed an increase in the length and number of endothelial filopodia, both at the tips and in the stalks of the sprouting vessels (Figure 2, A and B). Furthermore, consistent with the endothelial PlexinD1 distribution, numerous filopodia ectopically projected from the preformed capillaries around veins, but not arteries (Figure 2A). Thus, ECs, be they at the tips or in the stalks of sprouting retinal vessels, can elongate filopodia, and this is constitutively suppressed by PlexinD1 signaling. To further examine the contribution of PlexinD1 signaling to retinal vascular patterning, we examined the P5 retinal vasculature 48 hours after intravitreal PlexinD1-Fc injection at $\mathrm{P} 3$. This treatment led to the formation of a multilayered capillary network crossing over and under the veins (Figure 2A). By contrast, neutralization of endogenous VEGF rapidly depleted the endothelial filopodia throughout the developing retinal vasculature, which subsequently led to the abrogation of the capillary network (Figure 2, A and B). These results illustrate that the fine-tuning of endothelial filopodia regulated by VEGF and PlexinD1 signaling is a prerequisite for organizing the retinal vascular architecture.

Neuron-derived Sema3E restricts vascular pathfinding in developing retinas. Based on a previous study demonstrating identical vascular defects in mouse embryos deficient for the Plxnd1 and Sema3e genes (14), we next assessed the role of Sema3E in retinal vascular development. At P4, while the Plxnd1 gene was expressed in vascu- 

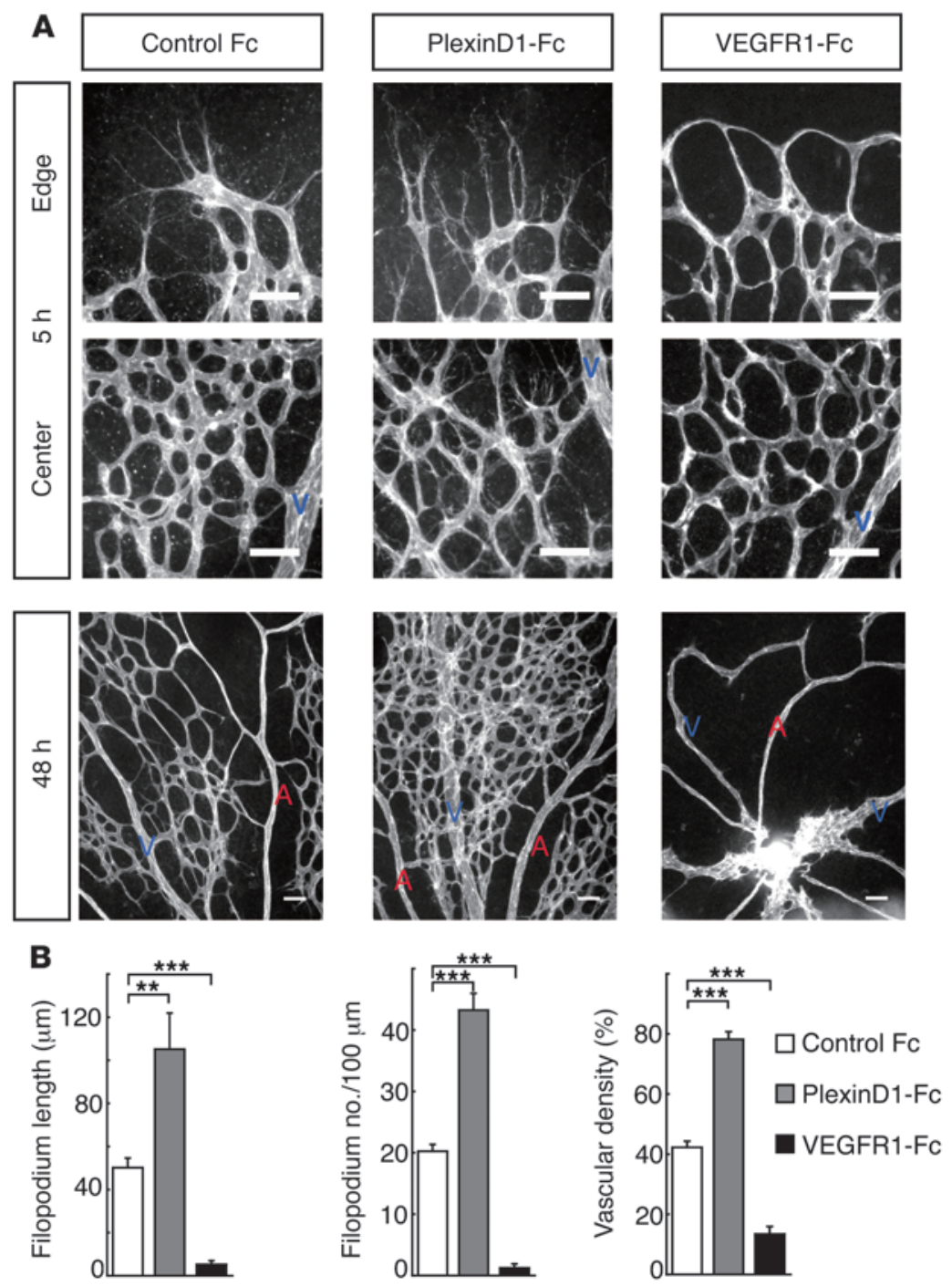

Figure 2

PlexinD1 signaling retracts endothelial filopodia in retinal vascular development. (A) Whole-mount PECAM-1 IHC in P5 retinas at 5 and 48 hours after intravitreal protein injection. PlexinD1-Fc rapidly induced elongation of endothelial filopodia in the sprouting vessels as well as in preformed capillaries, leading to the formation of a multilayered capillary network around veins. By contrast, VEGFR1-Fc depleted endothelial filopodia throughout the developing retinal vasculature, leading to the formation of a capillary-free vasculature. Scale bar: $50 \mu \mathrm{m}$. (B) Quantification of filopodium length ( $n=4$ per group) and filopodium number/100 $\mu \mathrm{m}$ vessel length ( $n=7$ per group) at the sprouting vascular fronts 5 hours after intravitreal injection, and vascular density $(n=4$ per group) around veins 48 hours after intravitreal injection. Error bars represent SEM; ${ }^{* * *} P<0.001,{ }^{* *} P<0.01$.

lar ECs and in a small subset (7.4\%) of underlying neurons in the ganglion cell layer (GCL), the Sema3e gene was expressed in $37 \%$ of neurons throughout the GCL (Figure 3A). Intravitreal injection of the Sema3E-alkaline phosphatase (Sema3E-AP) fusion protein, which can bind directly to PlexinD1 (14), identified its targets on the ECs of growing retinal vessels (Figure 3B). Because PlexinD1 is a primary receptor for Sema3E (14), these findings indicate that neuron-derived Sema3E acts on the overlying ECs expressing PlexinD1. In P4 retinas of Sema3e-deficient (Sema3e $\left.e^{--}\right)$mice, the retinal vascular patterning displayed a wide variety of morpho- logical defects, including solitary clusters of retinal ECs isolated from the advancing vascular fronts, attachment of hyaloid vessels to the retinal surface, and disorganized remodeling into distinct arteries, veins, and capillaries (Figure $3 \mathrm{C}$ and Supplemental Figure 2). While the length of endothelial filopodia was significantly increased in Sema3e $e^{-/-}$mice compared with that in Sema3e $e^{+/-}$mice, the overall vascular density was significantly decreased in Sema3 $e^{-/-}$retinas (Figure $3 \mathrm{D}$ ), which seems inconsistent with that seen in $\mathrm{P} 5$ retinas injected with the PlexinD1-Fc protein at P3 (Figure 2, $A$ and $B$ ). Nonetheless, the vascular phenotypes in $\mathrm{Sema}^{-/-}$retinas were reproduced by PlexinD1-Fc injection at P1 (Supplemental Figure 2I), which neutralized endogenous ligands for PlexinD1 throughout the entire process of retinal vascular development. Of note, we observed ectopic vascular invasion into the GCL of Sema3e $e^{-/}$mice (Figure 3E), suggesting that diffusion of Sema3E prevents misdirected vertical projections of endothelial filopodia, thereby facilitating the horizontal extension of the monolayered vascular network in the superficial retinal area (Figure $3 \mathrm{~F}$ ).

Sema3E-induced activation of the small GTPase RhoJ retracts endothelial filopodia. To determine the molecular mechanisms underlying the counteracting property of Sema3E-PlexinD1 signaling in terms of VEGF-induced filopodia projections, we sought out endothelial Rho small GTPases, intracellular molecular switches for the cytoskeletal rearrangements that comprise more than 20 different proteins (21-23). By performing microarray gene expression analyses exploiting ECs sorted from neonatal mouse retinas in conjunction with wholemount retinal in situ hybridization (ISH), we found that the Rhoj gene (also known as Tc10-like [Tcl], ref. 24 , and previously referred to as Rhot; ref. 25) was expressed almost exclusively in ECs in developing retinas (Figure 4A). Interestingly, endothelial Rhoj expression was prominent in veins and capillaries of growing retinal vessels, but was downregulated at later postnatal stages, which synchronized with the Plxnd1 expression (Supplemental Figure 3). Based on its protein structure, RhoJ is categorized into the Cdc42 small GTPase subfamily $(26,27)$, and it was previously reported that RhoJ induces filopodia projections in cultured neuronal cells (25). However, in cultured HUVECs, ectopic expression of the wild-type RhoJ (RhoJ-WT) and its constitutively active form (RhoJ-CA), but not the dominant-negative form (RhoJ-DN), induced cell contraction with reduction of stress fibers (Figure 4, B and D, and Supplemental Figure 4A; see also Supplemental Video 1). Of note, ectopically expressed RhoJ-WT proteins accumulated in the membrane blebs, to which endogenous RhoJ proteins were also localized after Sema3E stimulation (Supplemental Figure 4B). This contrasts with the cytoplasmic localization of RhoJ proteins in normal or VEGF-stimulated HUVECs (Supplemental Figure 4B). Intriguingly, Sema3E-induced cell contraction was abolished by knockdown of RhoJ with siRNA 

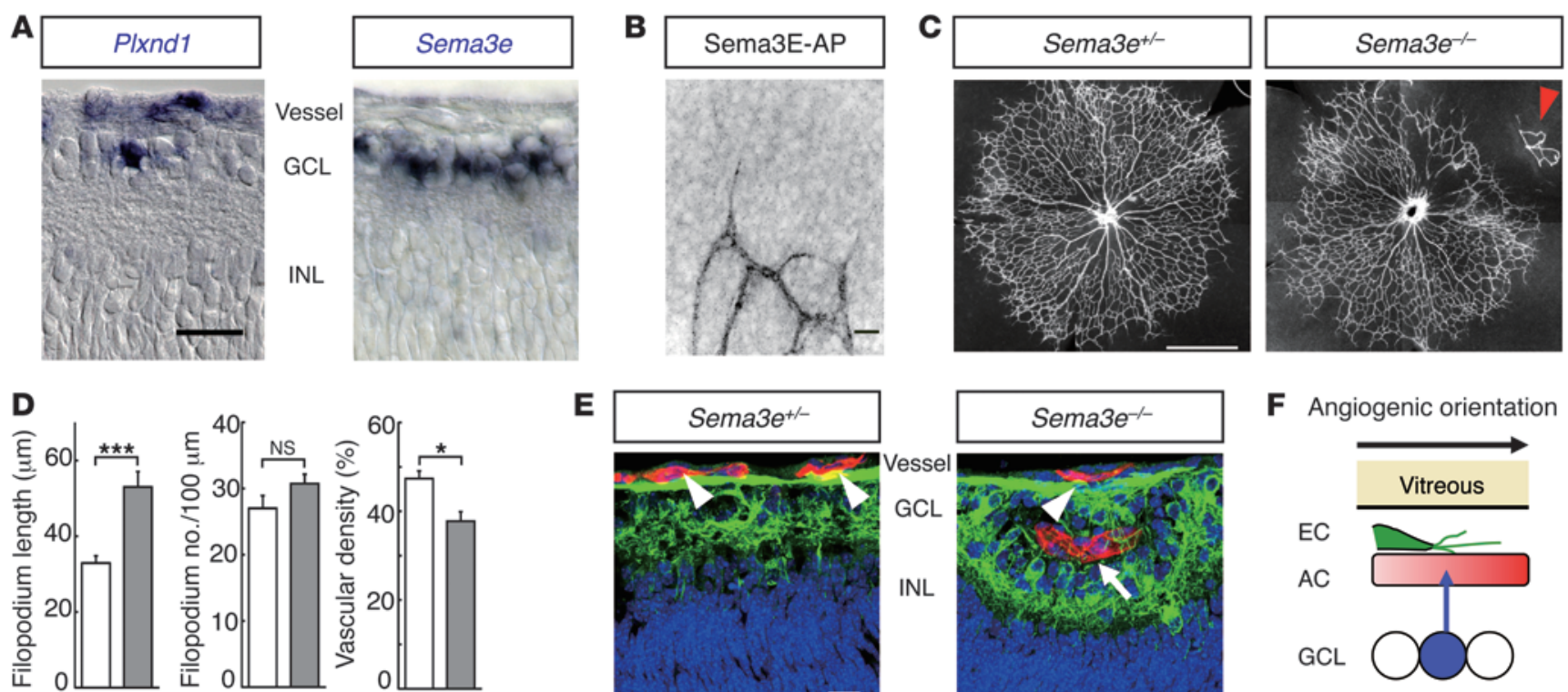

$\square$ Sema3e $e^{+/-}$

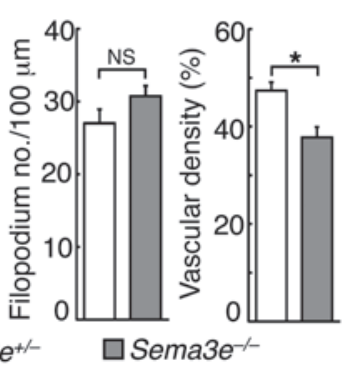

E

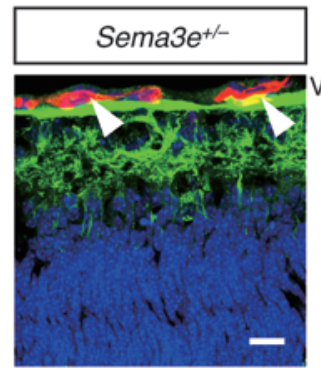

$\mathbf{F}$

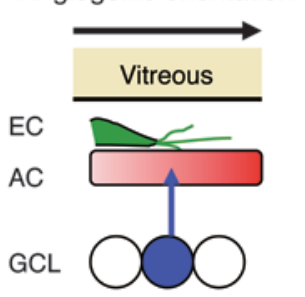

Figure 3

Neuron-derived Sema3E restricts vascular pathfinding in developing retinas. (A) ISH in P4 retinal sections. PIxnd1 is expressed in vascular ECs and in $7.4 \%$ of the neuronal populations in the GCL. Sema3e is expressed in $37 \%$ of neurons in the GCL. INL, inner nuclear layer. (B) High-magnification image of the advancing vascular front in AP-stained retina after intravitreal injection of Sema3E-AP fusion protein. (C) Whole-mount PECAM-1 IHC in P4 retinas. In the Sema3e-/- retina, the vascular network is disorganized in the upper-right quadrant. Note the attachment of hyaloid vessels to the retinal surface (arrowhead). (D) Quantification of filopodium length ( $n=18$ per group) and filopodium number/100 $\mu \mathrm{m}$ vessel length ( $n=7$ per group) at the sprouting vascular fronts, and vascular density ( $n=4$ per group) in P4 Sema3e-deficient retinas. Error bars represent SEM; ${ }^{* \star} P<0.001,{ }^{\star} P<0.05$. (E) Triple labeling for neurofilaments (green), PECAM-1 (red), and nuclei (blue) in retinal sections of P4 Sema3e-deficient mice. The superficial vessels (arrowheads) over the neuronal axons ectopically invade the GCL of Sema3e-l- mice (arrow). (F) A schematic diagram representing VEGF-expressing astrocyte (AC, red), PlexinD1-expressing EC (green), and Sema3E-expressing neuron (blue) in the superficial area of the developing mouse retina. Scale bars: $20 \mu \mathrm{m}$ (A, B, and E); $100 \mu \mathrm{m}$ (C).

(Figure 4, C and D, and Supplemental Figure 4C; see also Supplemental Videos 2-4), indicating that RhoJ is a pivotal intracellular molecule that mediates Sema3E-induced retraction of endothelial filopodia. Indeed, RhoJ was inactivated by VEGF, but activation was restored by exposure to Sema3E (Figure 4E). Thus, RhoJ is the common downstream target of the VEGF and Sema3E signals in regulating endothelial cytoskeletal rearrangements (Figure 4F).

To further evaluate the role of RhoJ in retinal vascular development, we used a gain-of-function gene-targeting approach. For this purpose, we inserted a cDNA encoding the murine wild-type Rhoj gene, preceded by the synthetic CAG promoter (28) and a loxP-flanked $\mathrm{Neo}^{\mathrm{r}}$-STOP cassette, into the ubiquitously expressed Rosa26 locus (ref. 29 and Supplemental Figure 5A). We crossed these conditional $\mathrm{Tg}$ mice carrying the allele termed R26-Rhoj to Tie2Cre Tg mice (30) to obtain offspring overexpressing RhoJ in ECs. After mating Tie2Cre ${ }^{T_{g} / W T} R 26-R h o j{ }^{T_{g} / W T}$ male with $R 26-R h o j{ }^{T_{g} / T_{g}}$ female mice, we could not obtain Tie2Cre ${ }^{T_{g} / W T} R 26-\mathrm{Rhoj}^{\mathrm{T}_{g} T_{\mathrm{g}}}$ neonates (Supplemental Figure 5B), indicating their embryonic lethality because of endothelial RhoJ overexpression. Indeed, $\mathrm{Tie}_{2} \mathrm{Cr} \mathrm{e}^{\mathrm{T}_{\mathrm{S}} / \mathrm{TT}}$ $R 26-\mathrm{Rhoj}^{\mathrm{Tg} / \mathrm{Tg}}$ embryos at $10.5 \mathrm{dpc}$ demonstrated growth retardation, with defective vascular development (Supplemental Figure 5C). We therefore attempted to analyze retinal vascular development in Tie2Cre ${ }^{T_{g} / W T} R 26-R h o j^{T_{g}}{ }^{W T}$ neonates, in which endothelial RhoJ overexpression was monitored by the bicistronic expression of EGFP upon excision of the Neor-STOP cassette (Figure 4G and Supplemental Figure 5D). Consistent with the ectopic RhoJ expression in cultured HUVECs, we observed numerous membrane blebs formed in ECs of sprouting retinal vessels (Figure 4H). This was also observed in inducible RhoJ overexpression in ECs after systemic administration of 4-hydroxytamoxifen (4OHT) to CAG-MerCreMer mice (31) carrying the R26-Rhoj allele (Figure 4I). Because endothelial filopodia projections were not apparently affected in RhoJ-overexpressing ECs, the membrane blebbing implied the fast cycling of projection and retraction of endothelial filopodia.

Extraretinal vessels highly express PlexinD 1 and RhoJ in ischemic retinas. With the molecular machinery underlying the intraretinal angiogenesis in mind, we next attempted to unveil the etiological process of extraretinal angiogenesis in ischemic retinopathies. For this purpose, we examined the spatial distribution of cells expressing Vegf, Sema3e, Plxnd1, and Rhoj genes in a mouse model of oxygeninduced retinopathy (OIR) (32). In this model, the mouse pups were housed in a hyperoxic state from P7 to P12, resulting in the regression of preformed capillaries in the central retinal areas (Supplemental Figure 6A). When the pups were returned to normal room air, the arising hypoxia induced vascular regrowth from the remaining vessels toward the ischemic retinas. At around P15, while retinal revascularization proceeded, new blood vessels protruded out of the retinal surfaces, as seen in human ischemic retinopathies. At P16, Veef expression was almost absent in astrocytes, unlike during development (Figure 5, A-C). Instead, a considerable number of the underlying neurons in ischemic retinal areas expressed Vegf. By contrast, Sema3e was broadly scattered across neurons throughout the GCL (Figure 5, D-F), as seen in normal retinas (Supplemental Figure 3). Intriguingly, the ECs of extraretinal 

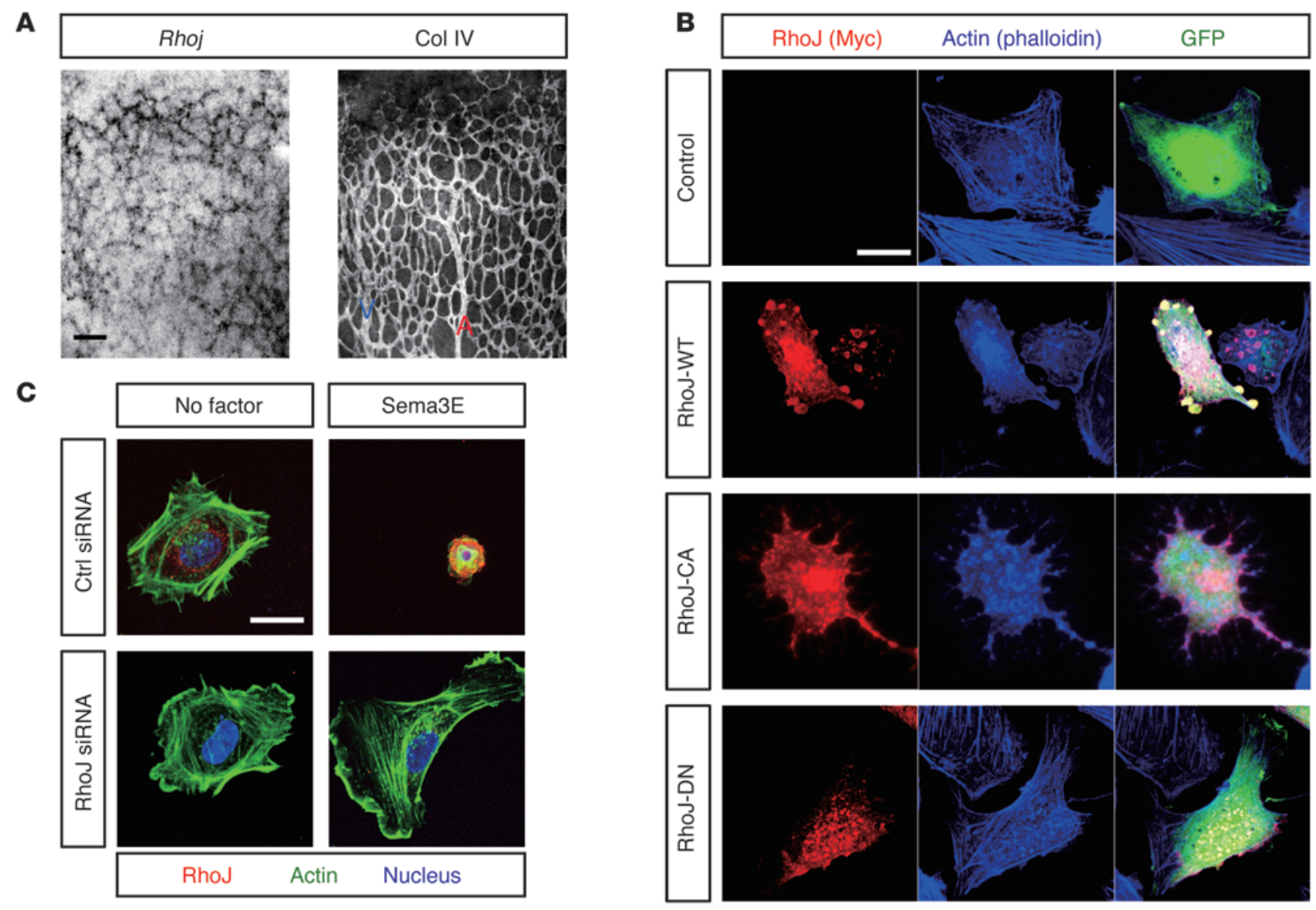

D Collapse/GFP Collapse/whole
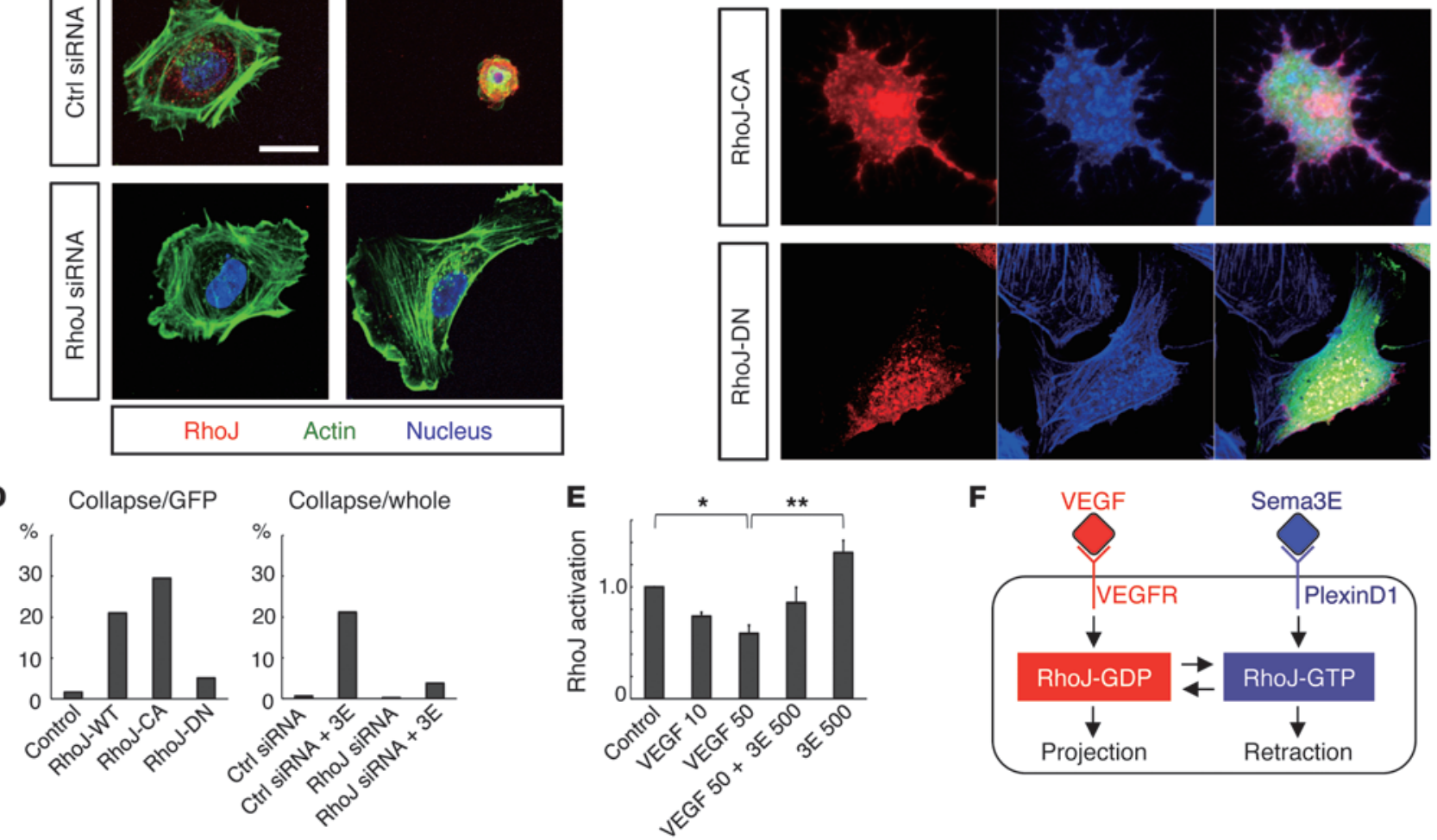

G
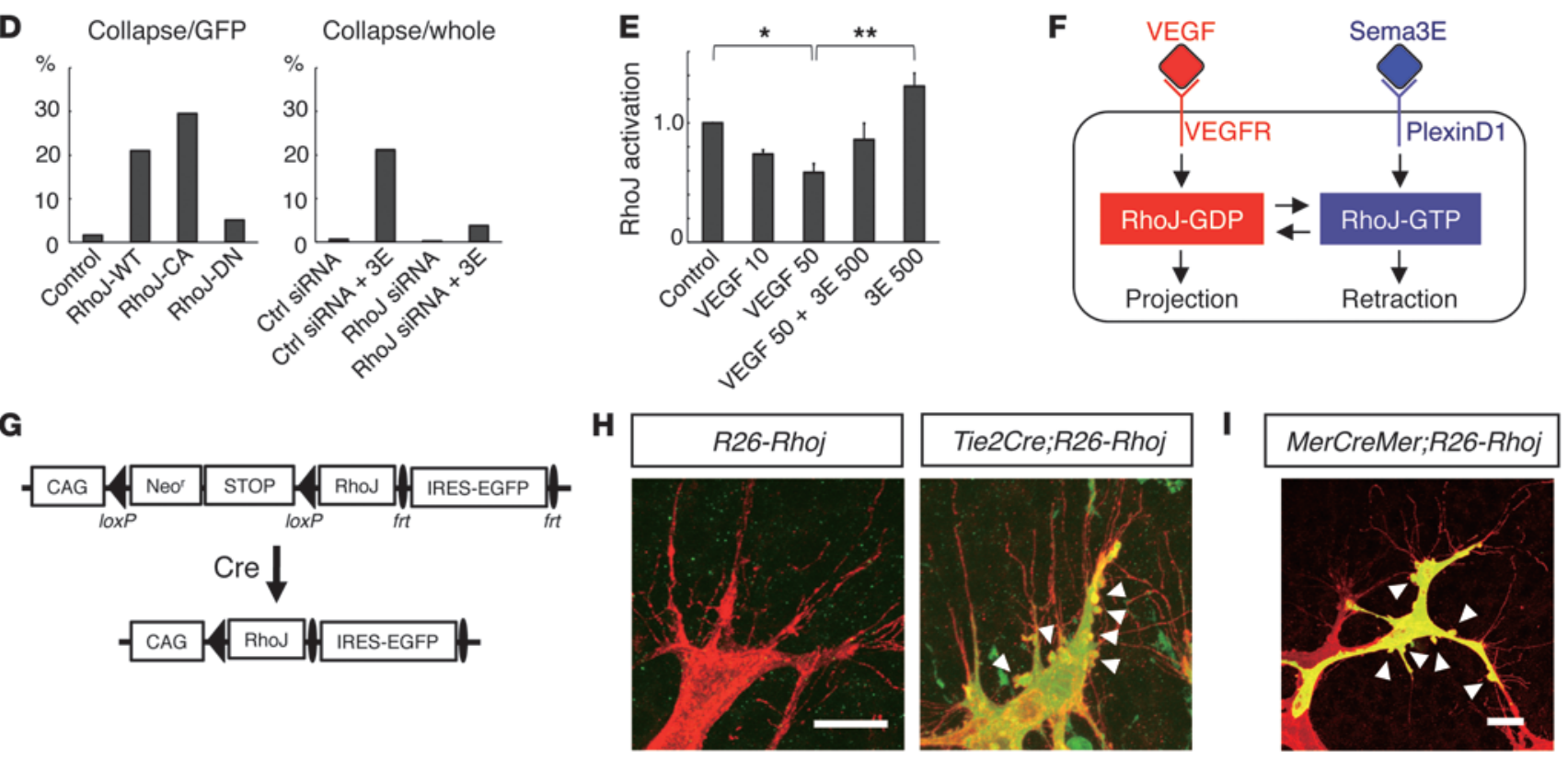


\section{Figure 4}

Sema3E-induced activation of the small GTPase RhoJ retracts endothelial filopodia. (A) Whole-mount ISH for Rhoj and IHC for Col IV in the P4 retina. (B) Contraction of cultured HUVECs by ectopic RhoJ expression. HUVECs were transfected with a vector expressing the Myc-tagged RhoJ protein, together with a GFP-expressing vector to identify transfected cells. Note the localization of RhoJ-WT proteins in the membrane blebs. (C) Abrogation of Sema3E-induced contraction of cultured HUVECs by knockdown of RhoJ. Note that targeted, but not control (Ctrl), siRNA led to the disappearance of immunoreactivity for RhoJ (red). (D) Proportions of collapsing cells relative to the GFPexpressing HUVECs in B (left) and to the whole siRNA-pretreated HUVECs in C (right). (E) Measurements of RhoJ activation in cultured HUVECs. RhoJ-GTP levels were diminished by VEGF but restored by Sema3E (3E). The values 10, 50, and 500 indicate the protein concentrations $(\mathrm{ng} / \mathrm{ml})$. Error bars represent SEM; ${ }^{\star \star} P<0.01$, ${ }^{\star} P<0.05$. (F) Schematic diagram representing RhoJ as an intracellular molecular switch mediating endothelial filopodia retraction. VEGF and Sema3E inversely regulate the activation status of RhoJ. (G) Scheme of the R26-Rhoj Tg. (H and I) Whole-mount IHC for PECAM-1 (red) and GFP (green) in P5 (H) and P7 (I) retinas of R26-Rhoj conditional Tg mice. In I, 4OHT was systemically administered to the CAG-MerCreMer;R26-Rhoj double $\mathrm{Tg}$ mice at P3. Note the membrane blebbing (arrowheads) in RhoJ-overexpressing ECs revealed by GFP expression at the sprouting vascular fronts. Scale bars: $100 \mu \mathrm{m}(\mathbf{A}) ; 20 \mu \mathrm{m}$ (B, C, H, and I).

vessels highly expressed the Plxnd1 gene, while its expression was hardly detectable in intraretinal vessels (Figure 5, G-I). Likewise, prominent $R h o j$ expression was limited to the extraretinal vessels (Figure 5, J-L). These findings suggest that neuron-derived Sema3E signals to endothelial PlexinD1 and activates RhoJ restrictively in extraretinal vessels.

PlexinD1 signaling suppresses extraretinal vascular outgrowth in ischemic retinas. Because neuron-derived Sema3E prevents downward vascular growth in developing retinas, we suspected that diffusion of Sema3E through the superficial retinal areas might force the PlexinD1-expressing ECs out of the retinal surfaces in the OIR model (Figure 6A). Unexpectedly, however, 24 hours after intravitreal injection of PlexinD1-Fc at P14, we observed extraretinal projections of numerous filopodia (Figure 6B). By contrast, VEGFR1-Fc injection completely abolished extraretinal vascular outgrowth, while discrete fragments of intraretinal ECs implied progressive vascular regression (Figure 6B).

To further assess the roles of VEGF and PlexinD1 signaling in the construction of the disorganized vascular architecture in the OIR model, we inactivated the genes Vegfr 2 and Plxnd1, encoding endothelial receptors, using tamoxifen-inducible Cre-loxP-mediated genetic recombination (Supplemental Figure 6, B and C). After daily systemic administration of $4 \mathrm{OHT}$ to CAG-MerCreMer mice carrying loxP-flanked (33) and constitutive null (34) alleles for the Vegfr2 (Vegfr flox/-) gene, we observed complete depletion of the extraretinal vessels (Figure 6, C and D). Concurrently, the intraretinal vascular regeneration was also interrupted, leading to a substantial decrease in capillary density, with large unvascularized retinal areas (Supplemental Figure 7). By contrast, inducible inactivation of the Plxnd1 gene $(14,35)$ led to a conspicuous increase in the number of extraretinal vessels, while intraretinal vascular regeneration proceeded as in the control retina (Figure 6, C and D). Consistent with the notion that RhoJ acts as a downstream molecule of PlexinD1 signaling, inducible RhoJ overexpression in the OIR mice resulted in a significant decrease in the number of extraretinal vessels, while intraretinal capillary density was also reduced (Figure 6, C and D). These results together indicate that PlexinD1 signaling protects blood vessels in ischemic retinas from VEGF-induced extraretinal vascular outgrowth.

Intravitreal Sema3E protein restores proper angiogenic orientation in ischemic retinas. Based on the findings described above, we hypothesized that diffusion of neuron-derived Sema3E into the preretinal space might act on extraretinal PlexinD1 but is insufficient to fully suppress VEGF-induced disoriented angiogenesis in ischemic retinopathy. Therefore, to directly stimulate PlexinD1 in the extraretinal vessels, we injected Sema3E protein into the vitreous space at P14 in the OIR model. At 24 hours after injecting $1 \mu \mathrm{l}$ of Sema3E protein at a concentration of $2 \mu \mathrm{g} / \mu \mathrm{l}$, we found no apparent effects on intraretinal filopodia projections (Figure 7A), which was attributed to the lack of PlexinD1 expression in these ECs. However, at 72 hours after this treatment, we observed a significant decrease in extraretinal vessels (Figure 7, B and C). Notably, this effect was more pronounced, without reducing intraretinal capillary density, after injection of Sema3E protein at a higher concentration (Figure 7C and Supplemental Figure 8). The newly formed intraretinal vessels were almost uniformly associated with periendothelial mural cells (Supplemental Figure 9). Thus, the intravitreal Sema3E injection led to the regeneration of the normal vascular architecture by restoring angiogenic orientation in ischemic retinas. By contrast, VEGFR1-Fc injection suppressed the intraretinal projections of endothelial filopodia (Figure 7A), with concomitant regression of the remaining capillaries (Supplemental Figure 9), which resulted in extensive ischemic retinal areas (Figure 7, B and C, and Supplemental Figure 8).

\section{Discussion}

Anti-VEGF drugs have been reported to temporarily halt disease progression in some refractory cases of ischemic retinopathies $(5,6)$. This pharmacological approach has offered a breakthrough that obviates destructive therapies such as laser photocoagulation and surgical interventions. However, as shown in our study, comprehensive inhibition of angiogenesis may sustain retinal ischemia, which will consequently exacerbate disordered angiogenesis unless the anti-VEGF drugs are repeatedly administered. To overcome this limitation, we postulated that normalization of angiogenic orientation will offer an alternative, but effective treatment to fundamentally resolve retinal ischemia. Starting from this premise, we explored a therapeutic approach that can selectively suppress disoriented angiogenesis by exploiting the molecular mechanisms underlying intraretinal angiogenesis during development.

In postnatal mouse retinas, we observed an intimate association between PlexinD1-expressing ECs and VEGF-expressing astrocytes, which was corroborated by Plxnd1 gene downregulation after blocking VEGF signaling. While blocking PlexinD1 signaling rapidly elongated endothelial filopodia, the disruption of Sema3e, which is expressed in neurons in the GCL, caused defective patterning of the retinal vasculature. Based on these findings, we propose that Sema3E-PlexinD1 signaling acts as a negative feedback system against VEGF signaling in terms of the filopodia projections as follows: Under hypoxia, VEGF evokes a series of proangiogenic signaling cascades for proliferation, survival, and migration (36) but simultaneously upregulates PlexinD1 expression. Sema3E diffusing from the underlying neurons constantly signals to endothelial PlexinD1. ECs can project filopodia when VEGF signaling rises above the threshold level defined by the PlexinD1 signaling. 

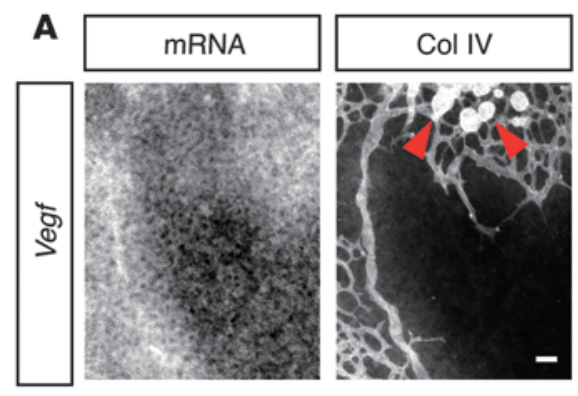

D
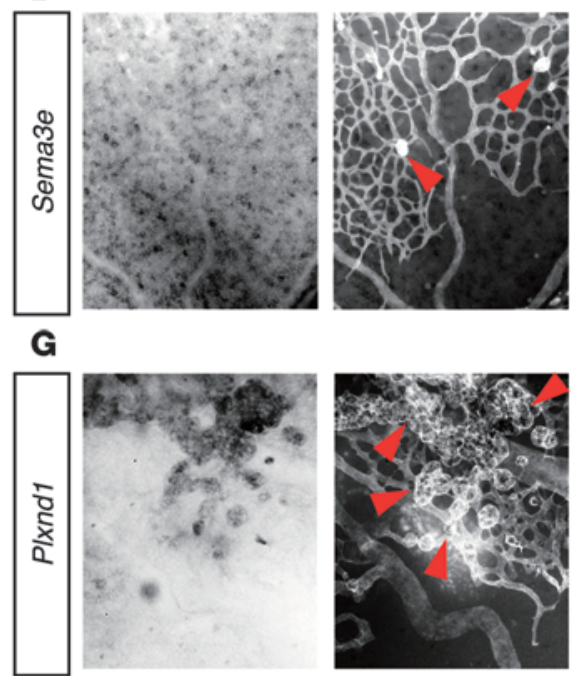

J
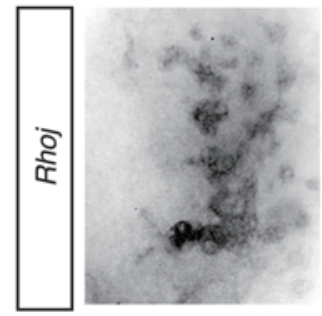

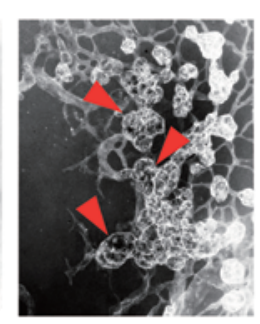

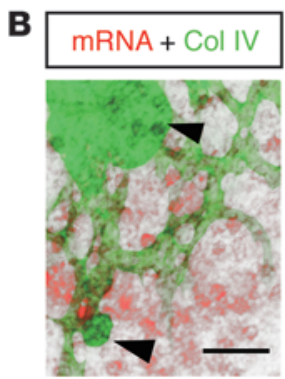

E

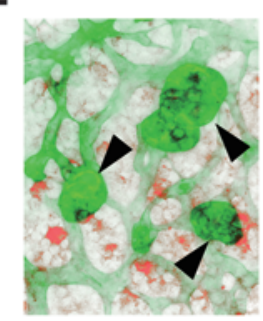

H

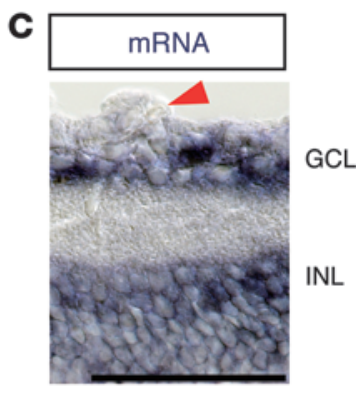

$\mathbf{F}$

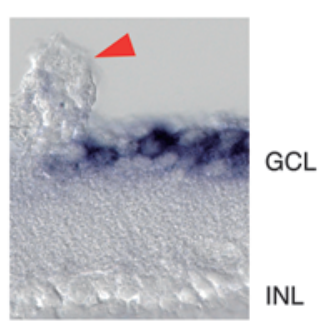

I

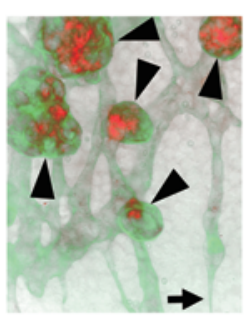

$\mathbf{K}$

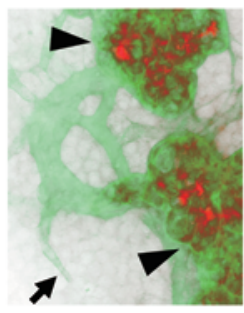

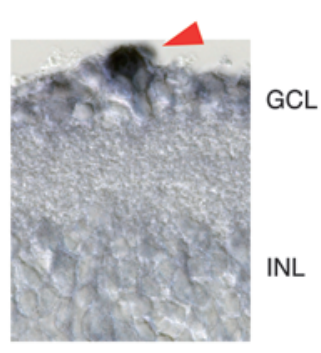

$\mathbf{L}$

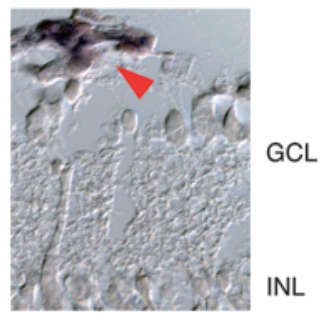

\section{Figure 5}

Extraretinal vessels highly express PlexinD1 and RhoJ in ischemic retinas. (A, D, G, and J) Whole-mount ISH in conjunction with IHC for Col IV in P16 OIR retinas. ( $\mathbf{B}, \mathbf{E}, \mathbf{H}$, and $\mathbf{K}$ ) High magnification of merged images of ISH (red) and Col IV IHC (green). (C, F, I, and L) ISH in sections of P16 OIR retinas. Neurons in the GCL and INL strongly express Vegf in the ischemic retinal areas $(\mathbf{A}-\mathbf{C})$, whereas Sema3e is expressed in neurons scattered throughout the GCL (D-F). Endothelial expression of the PIxnd1 (G-I) and Rhoj (J-L) genes is prominent in extraretinal vessels, but is hardly detectable in intraretinal vessels. Arrowheads, extraretinal vessels; arrows, advancing vascular fronts of intraretinal vessels. Scale bars: $50 \mu \mathrm{m}$.
In the present study, we showed for the first time to our knowledge that Sema3E-induced activation of RhoJ mediates endothelial contraction. This contrasts with VEGF-induced activation of Cdc42 (37), which plays a crucial role in filopodia projections $(26,27)$. Interestingly, RhoJ and Cdc42 were inactivated by VEGF and Sema3E, respectively (Supplemental Figure 10A). Thus, it seems likely that VEGF and Sema3E signals inversely activate or inactivate $\mathrm{Cdc} 42$ and RhoJ. Upon activation, both RhoJ and $\mathrm{Cdc} 42$ bind to the $\mathrm{Cdc} 42 / \mathrm{Rac}$-interactive binding (CRIB) domains of their effector proteins, such as p21-activated kinase (PAK) and neural Wiskott-Aldrich syndrome protein (N-WASP), which regulate intracellular actin polymerization $(24,25)$. Given the opposite effects of RhoJ and Cdc42 on endothelial filopodia formation, we suggest that activated RhoJ and Cdc42 competitively bind to their effector proteins, whereby the endothelial filopodia project or retract (Supplemental Figure 10B). Considering the expression of $\mathrm{Cdc} 42$ in various types of cells, the endothelial expression of RhoJ indicates the possibility that this molecule could serve as a therapeutic target to specifically manipulate endothelial filopodia projections.
Given the molecular machinery described above, one may ask how such a fine-tuning of endothelial filopodia projections contributes to the establishment of the retinal vascular architecture. A key to answering this question may be the contradictory consequences of filopodia elongation after PlexinD1-Fc injections at different time points. PlexinD1-Fc injection at P3 led to the formation of an excessive capillary network around veins, whereas its injection at P1 resulted in a sparse capillary network with less apparent morphological distinction between arteries and veins. This discrepancy may partly be explained by the extent of preformed vessels at the time of PlexinD1-Fc injection. That is, elongated filopodia may fuse extensively with neighboring vessels after P3, when the retinal vascular network has been considerably formed. By contrast, when PlexinD1 signaling is blocked just after the onset of the retinal vascular development, the aberrantly projected filopodia may fail to anastomose with each other, leading to the formation of a discontinuous capillary network. In line with this idea, the overall vascular density was decreased in Sema3edeficient retinas, despite the increased length of endothelial filopodia. Of importance, the Sema3e-deficient retinas demonstrated 
A

C
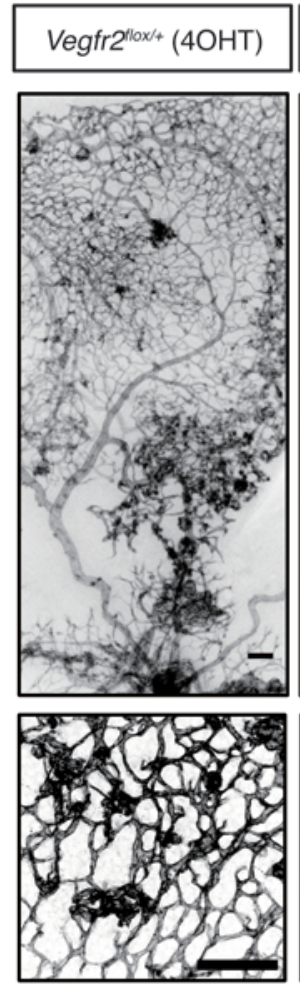

D

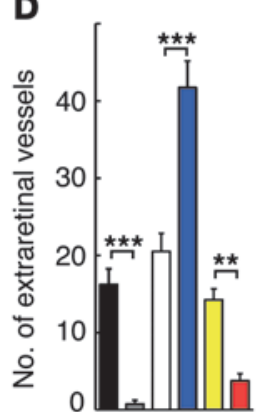

GCL
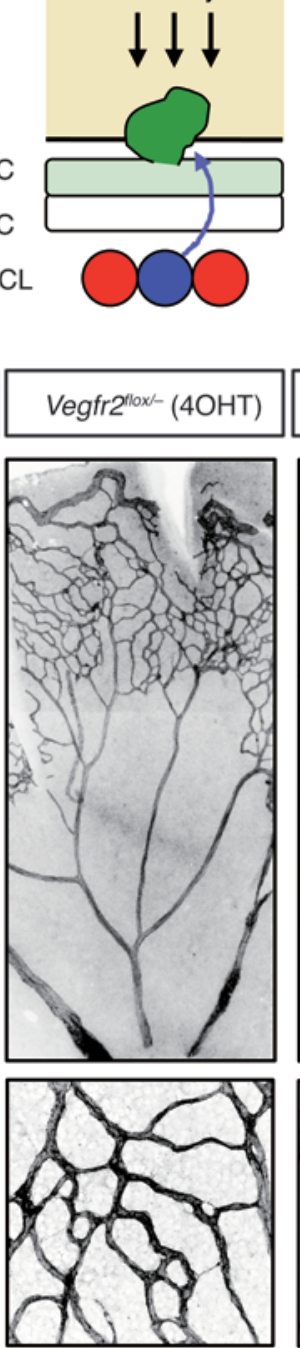

B

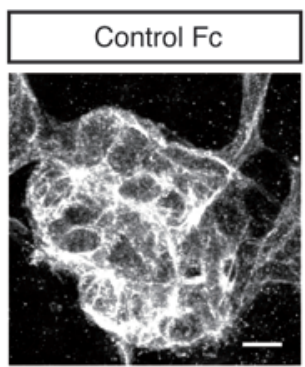

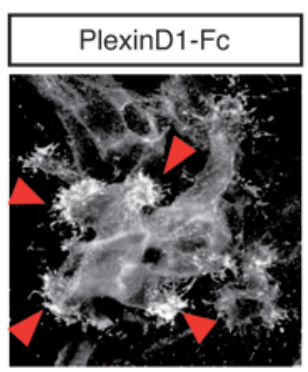

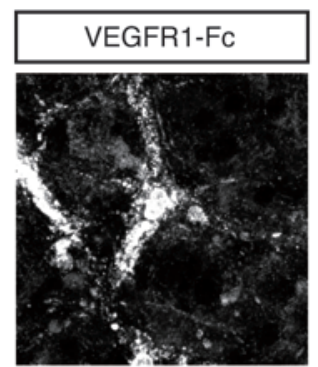

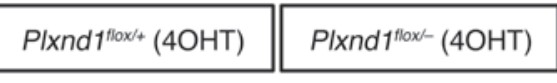

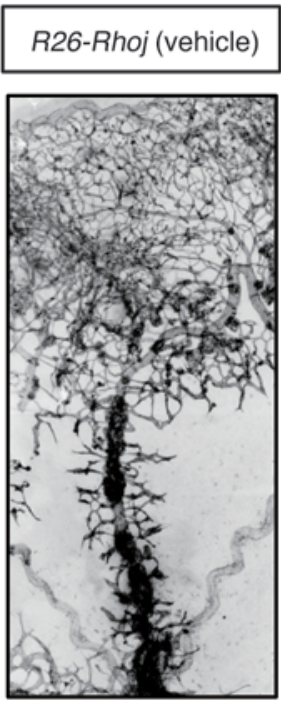

R26-Rhoj (4OHT)
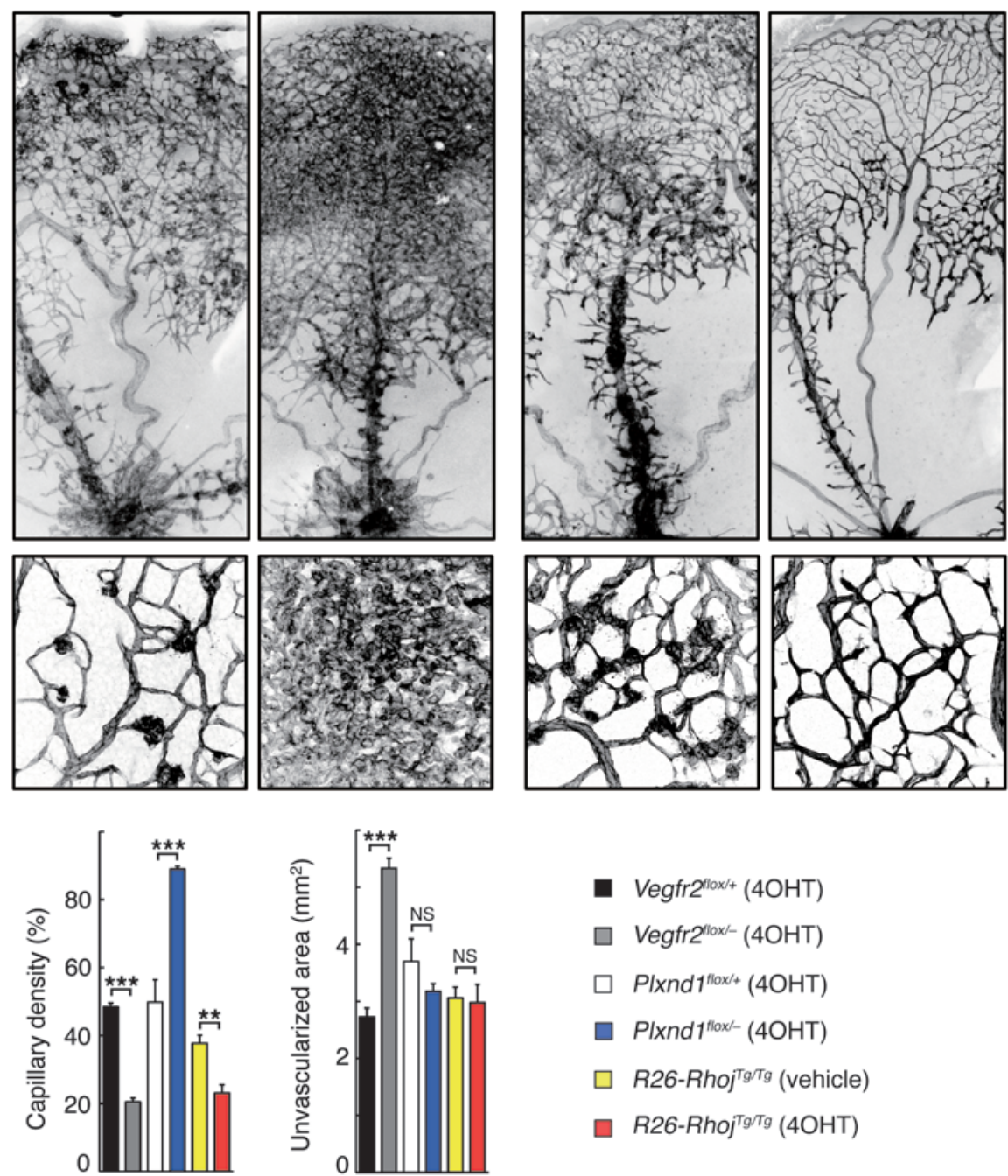

Figure 6

PlexinD1 signaling counteracts VEGF-induced extraretinal vascular outgrowth in ischemic retinas. (A) A schematic diagram representing VEGFexpressing neurons (red), PlexinD1-expressing extraretinal EC (green), and Sema3E-expressing neuron (blue) in the OIR model. (B) Wholemount PECAM-1 IHC in P15 OIR retinas 24 hours after intravitreal protein injection. PlexinD1-Fc induced extraretinal projections of endothelial filopodia (arrowheads). By contrast, VEGFR1-Fc abolished extraretinal vascular outgrowth with discrete endothelial fragments. (C) Whole-mount PECAM-1 IHC in P18 OIR retinas after tamoxifen-inducible Cre-loxP-mediated genetic recombination. CAG-MerCreMer mice carrying Vegfr2flox, PIxnd1-flox, or R26-Rhoj alleles were i.p. injected with 4OHT daily from P12. For the control of CAG-MerCreMer;R26-RhojTg/Tg mice, vehicle (1:9 ethanol/sunflower oil) was injected. Scale bars: $10 \mu \mathrm{m}$ (B); $100 \mu \mathrm{m}$ (C). (D) Quantification of the number of extraretinal vessels, capillary density in the vascularized area, and unvascularized retinal area in P18 OIR mice. Error bars represent SEM; ${ }^{* \star *} P<0.001,{ }^{* \star} P<0.01$. 


\section{A}
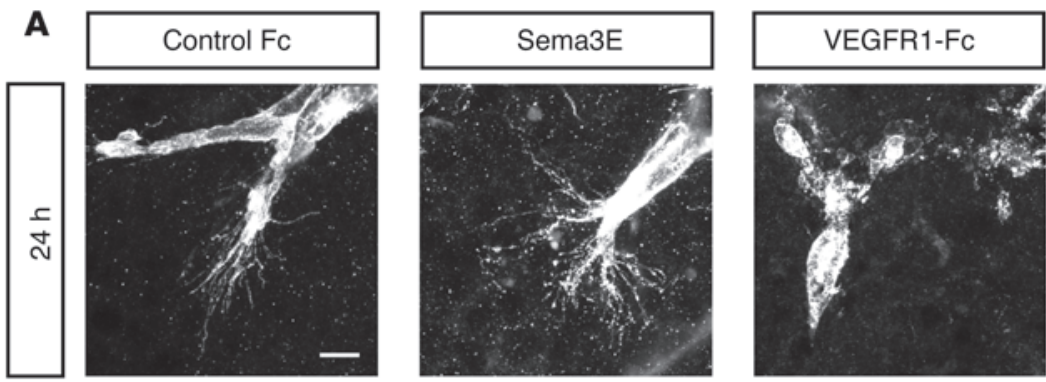

B
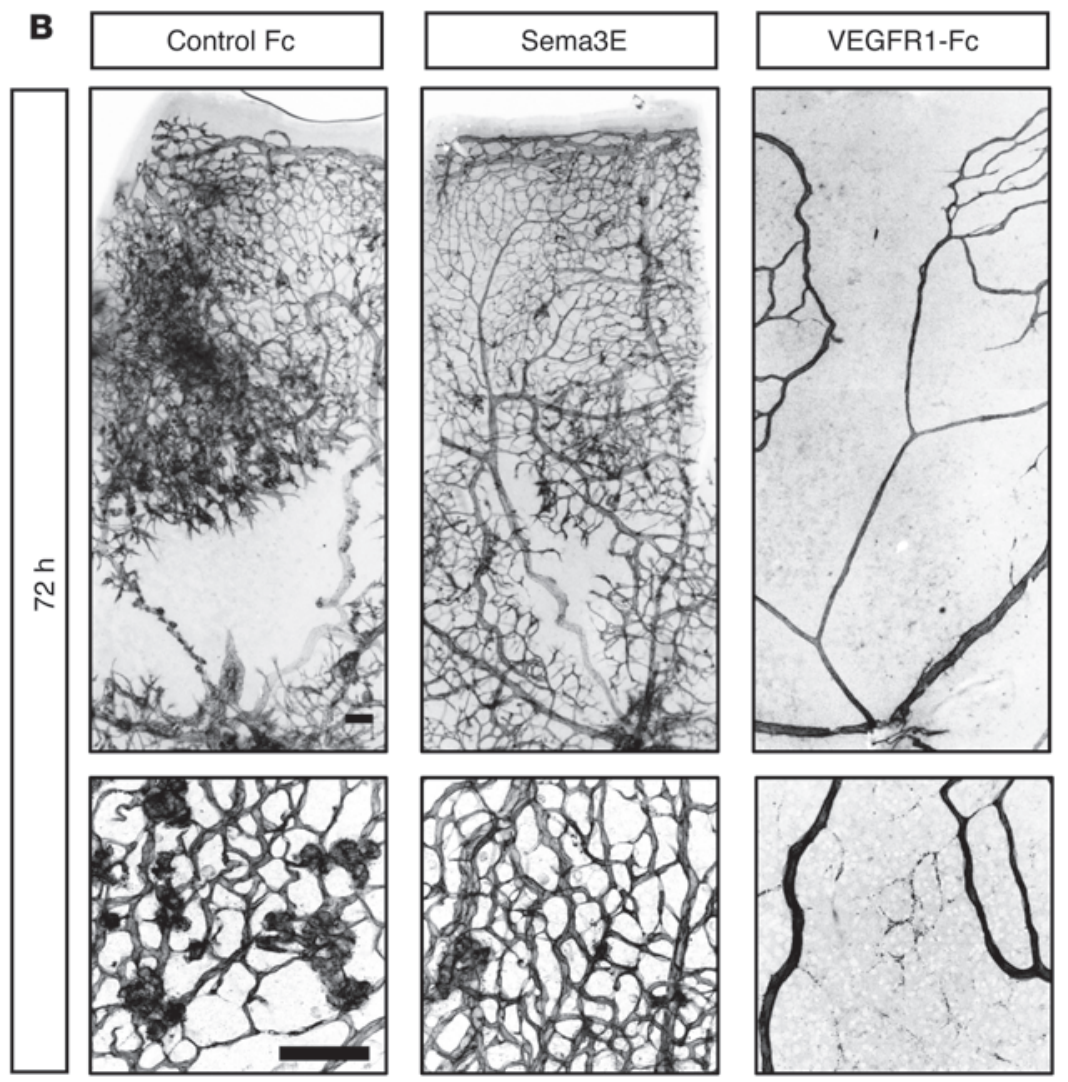

C
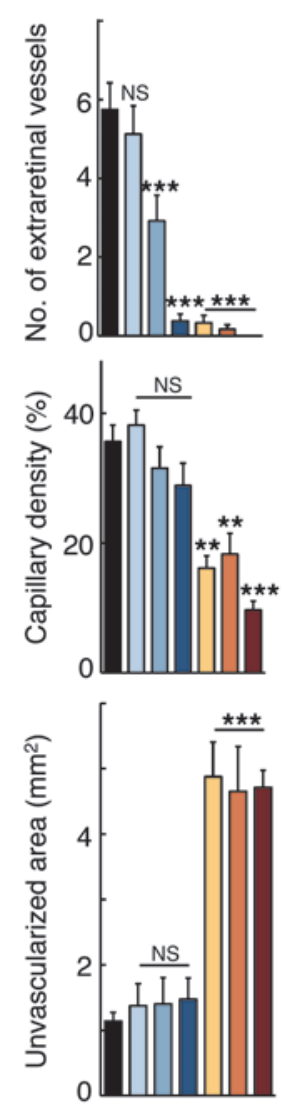

- Control Fc (2)

$\square$ Sema3E (1) $\square$ VEGFR1-Fc (1)

$\square$ Sema3E (2) $\square$ VEGFR1-Fc (2)

- Sema3E (4) घ VEGFR1-Fc (4)

Figure 7

Intravitreal Sema3E injection selectively suppresses extraretinal vascular outgrowth without affecting vascular regeneration in ischemic retinas. (A and B) Whole-mount PECAM-1 IHC in OIR retinas of C57BL/6 mice at 24 hours (A) and 72 hours (B) after intravitreal injections of recombinant proteins $(1 \mu \mathrm{l}$ volume at $2 \mu \mathrm{g} / \mu \mathrm{l}$ concentration) at P14. Sema3E did not affect intraretinal projections of endothelial filopodia at P15 (A), but did suppress extraretinal vascular outgrowth at P17, leading to the restoration of normal vascular architecture in ischemic retinas (B). VEGFR1-Fc completely suppressed endothelial filopodia projections (A), resulting in extensive ischemic retinal areas (B). Scale bars: $10 \mu \mathrm{m}(\mathbf{A}) ; 100 \mu \mathrm{m}$ (B). (C) Quantification of the number of extraretinal vessels, capillary density in the vascularized area, and unvascularized retinal area at 72 hours after injection of $1 \mu \mathrm{l}$ recombinant proteins at P14. The protein concentrations $(\mu \mathrm{g} / \mu \mathrm{l})$ are indicated in the parentheses. $P$ values were calculated by comparison with the Control Fc. Error bars represent SEM; ${ }^{\star \star \star} P<0.001,{ }^{\star \star} P<0.01$.

a wide variety of vascular defects in their extent as well as in their morphological phenotypes, which is ascribable to the functional redundancy of endogenous ligands for PlexinD1. While Sema3E is the only ligand that can directly bind to PlexinD1, other soluble Sema3 ligands may signal to PlexinD1 in the co-presence of neuropilins (38), to compensate for the loss of Sema3E or to exert their distinct functions.

In postnatal mouse retinas, developing vessels grow without protruding out of the retinal surface, even after pharmacological blockade of PlexinD1 or genetic disruption of Sema3e. This excludes the possibility that a deficiency in Sema3E-PlexinD1 signaling alone is sufficient for extraretinal angiogenesis. By contrast, overexpression of soluble VEGF can cause extraretinal projections of endothelial filopodia in developing retinal vasculature (9). As we showed the complete suppression of extraretinal angiogenesis by pharmacological or genetic disruption of VEGF signaling in the OIR model, it seems likely that aberrant VEGF distribution is primarily responsible for extraretinal angiogenesis. Indeed, the predominant VEGF-expressing cells in the OIR model are neurons rather than astrocytes. In addition, astrocytes failed to deposit 
extracellular fibronectin matrices (Supplemental Figure 11), which may have led to the loss of physical scaffolds for migrating ECs and the inability to retain heparin-binding VEGF proteins within the retina. The resulting diffusion and accumulation of VEGF in the preretinal space, as documented in human ischemic retinopathies (39), may induce disoriented extraretinal vascular outgrowth. In this context, a high VEGF concentration accounts for the elevated PlexinD1 expression in extraretinal vessels.

By exploiting the differential distribution of PlexinD1 in the OIR model, we showed that intravitreal Sema3E injection selectively prevented extraretinal vascular outgrowth. Because of the absence of PlexinD1 expression in intraretinal vessels, this treatment did not interfere with vascular regeneration in ischemic retinas. To date, experimental manipulation of various signaling molecules, such as inducible nitric oxide synthase (40) and sphingosine 1phosphatase (41), has been demonstrated to successfully prevent or repair vascular abnormalities in OIR models. Nonetheless, it is intriguing that diffuse administration of soluble Sema3E proteins can correct the imbalance in the complex signaling machineries determining retinal angiogenic orientation. This simplicity may allow clinical adaptation of this treatment for vascular regeneration therapy in ischemic retinopathies.

The feasibility of intravitreal Sema3E therapy is further supported by the specific involvement of PlexinD1 signaling in angiogenic sprouting, which will lessen the undesirable effects on normal vascular functions. However, the expression of PlexinD1 in neurons, although only limited to a small population in the GCL, suggests that caution must be exercised regarding the direct effects of Sema3E on retinal neurons, especially in clinical use for premature infants, whose retinal neural networks may not have been fully established (42). Indeed, in Sema3e $e^{-/}$mice, we occasionally observed disordered axonal fibers in small retinal areas (Supplemental Figure 12), implying a role for Sema3E in axonal pathfinding in specific populations of retinal ganglion cells. While we demonstrated dose-dependent efficacy of intravitreal Sema3E injection in suppressing disoriented angiogenesis, its appropriate dosage remains to be determined to minimize the potential undesirable effects.

Further studies are needed to evaluate whether intravitreal Sema3E administration alone can offer vascular regeneration therapy in ischemic retinopathies, because this treatment did not reduce the unvascularized retinal areas in the OIR model. In some cases of human ROP, in which physiological retinal angiogenesis spontaneously recurs, it has been clinically reported that intravitreal anti-VEGF therapy paradoxically promotes physiological angiogenesis (6). Nevertheless, our experimental results demonstrated that VEGFR1-Fc injection, irrespective of Sema3E coinjection, comprehensively inhibited vascular regrowth in the OIR model (Supplemental Figure 8), indicating the clinical requirements for restricted use of anti-VEGF drugs in severe ROP. Also, in human PDR, which often lacks spontaneous vascular regeneration in ischemic retinas, selective suppression of extraretinal angiogenesis by Sema3E injection will be more beneficial when conjugated with additional modalities that promote intraretinal vascular growth, such as restoration of proangiogenic properties in retinal astrocytes (43).

In conclusion, we have presented a paradigm of vascular regeneration therapy in ischemic retinas by exploiting the antiangiogenic action of Sema3E on PlexinD1-expressing extraretinal vessels. Sema3E administration can also be useful to precisely guide angio- genesis to ischemic tissues in other organs if PlexinD1 expression is restricted to disoriented vessels. Otherwise, in diseases with broad PlexinD1 expression in abnormal vessels, such as cancer (44), Sema3E administration may be a potent antiangiogenic therapy. Thus, the outcomes of Sema3E therapy may depend on the distribution of endothelial PlexinD1 in distinct disease settings.

\section{Methods}

Mice. Analyses of retinal vascular development were performed in WT mice with ICR or C57BL/ 6 backgrounds (SLC Japan). For the generation of R26-Rhoj Tg mice (RIKEN CDB acc. no. CDB0919K; http://www.cdb. riken.jp/arg/mutant\%20mice\%20list.html), a cDNA encoding a native form of murine Rhoj was cloned into a modified version of pROSA26-1 vector (29) (provided by Y. Sasaki, RIKEN CDB), preceded by the ubiquitous CAG promoter (28) and a loxP-flanked $\mathrm{Neo}^{\mathrm{r}}$-STOP cassette, followed by a frt-flanked IRES-EGFP cassette and a bovine polyadenylation sequence (Supplemental Figure 5A). Gene targeting was done with TT2 ES cells and confirmed by Southern blotting. Mice were generated by injection of targeted ES cells into ICR embryos and backcrossed with the C57BL/6 strain. PCR genotyping was performed with forward primer R26F2 (5'-AAAGTCGCTCTGAGTTGTTAT-3') and reverse primer R1295 (5'GCGAAGAGTTTGTCCTCAACC-3') (product size of 303 bp for the R26Rhoj allele) and reverse primer R523 (5'-GGAGCGGGAGAAATGGATATG$3^{\prime}$ ) (product size of $601 \mathrm{bp}$ for the WT allele). Female mice carrying the R26-Rhoj allele were bred with Tie2Cre (30) male mice (provided by R. Wang, UCSF, San Francisco, California, USA), to delete the $\mathrm{Neo}^{\mathrm{r}}$-STOP cassette and drive RhoJ expression in ECs. Frozen embryos of Sema3e-deficient mice (14) with a C57BL/6 background (European Mouse Mutant Archive ID, EM:01373) were provided by C.E. Henderson, Columbia University, New York, New York, USA. CAG-MerCreMer Tg mice (31) (RIKEN CDB acc. no. CDB0404T) were mated with Plxnd1-deficient (14) and Plxnd1-floxed (35) (provided by T.M. Jessell, Columbia University, New York, New York, USA), Vegfr2-deficient (34) and Vegfr2-floxed (33) (provided by J. Rossant, Hospital for Sick Children Research Institute, Toronto, Canada), and R26-Rhoj mice with C57BL/ 6 backgrounds to obtain tamoxifen-inducible conditional mutants. Cre-loxP-mediated genetic recombination in the OIR model was induced by i.p. injections of $30 \mu \mathrm{lof} 10 \mathrm{mg} / \mathrm{ml} 4 \mathrm{OHT}$ (Sigma-Aldrich) dissolved in 1:9 ethanol/sunflower oil (Sigma-Aldrich) once daily from P12 after return to normal room air. The OIR model was established by maintaining the oxygen concentration at $75 \%$ by a ProOX oxygen controller (BioSpherix). All experimental protocols were reviewed and approved by the Institutional Animal Care and Use Committee of RIKEN CDB and Kobe University Graduate School of Medicine.

Histology. The procedures for ISH and immunohistochemistry (IHC) in whole-mount retinas have been described elsewhere (45). For IHC, retinas were incubated with the following primary Abs: polyclonal rabbit anti-type IV collagen (COSMO BIO Co.), monoclonal rat anti-PECAM-1 (clone Mec13.3; BD), polyclonal rabbit anti-neurofilament M (Millipore), and polyclonal rabbit anti-GFP (Invitrogen). The secondary Abs were Alexa Fluor 488-donkey anti-rabbit (Invitrogen) and Cy3-donkey antirat (Jackson ImmunoResearch Laboratories Inc.). Nuclear staining was performed using TO-PRO-3 iodide (Invitrogen). For ISH, the templates for cRNA probes (Vegf, 825-1,172 of NM_009505; Plxnd1, 5,184-6,055 of AK129175; Rhoj, 1,203-2,276 of NM_023275) were obtained by reverse transcription of total RNA from P4 ICR mouse retinas with subsequent PCR amplification. The templates for Sema3e RNA probes (46) were gifts from A. Watakabe (National Institute for Basic Biology, Okazaki, Japan). For cryosections, retinas were frozen in OCT compound (Sakura Finetek) after whole-mount ISH and sectioned at a thickness of $10 \mu \mathrm{m}$. Images were taken with an LSM510 confocal microscope (Zeiss), or with an Axioplan 2 
microscope (Zeiss) equipped with associated software (AxioVision, version 3.1; Zeiss). In combined ISH and IHC, photos of the same fields were taken separately in black and white with an Axioplan 2 microscope. The signals were then colored and merged using Adobe Photoshop CS software.

Intravitreal injections. PlexinD1-Fc protein was purified using a HiTrap Protein G column (GE Healthcare) from serum-free culture supernatants of FreeStyle 293F cells (Invitrogen) transfected with a plasmid vector expressing the extracellular domain of mouse PlexinD1 (Met1-Gly1266) fused to the Fc fragment of human IgG under control of the CAG promoter (20). Recombinant PlexinD1-Fc, mouse VEGFR1-human Fc (R\&D Systems), human Fc (Jackson ImmunoResearch Laboratories Inc.), and human Sema3E (R\&D Systems) proteins in sterile buffer were injected into the vitreous humor using glass capillary pipettes with a micromanipulator (Drummond Scientific Co.). In Sema3E-AP binding assays, the retinal cups were removed 2 hours after intravitreal injection of the Sema3E-AP fusion protein, washed, and stained as previously described (14).

Quantitative analysis of the retinal vasculature. All quantifications were performed in images of flat-mount retinas stained with PECAM-1. The number of endothelial filopodia per $100-\mu \mathrm{m}$ vessel length, and filopodia lengths were calculated in sprouting vascular fronts photographed at $63 \times$ objective using LSM510 confocal image software. The vessel area density in postnatal mouse retinas was calculated in a fixed area $\left(3.63 \times 10^{4} \mu \mathrm{m}^{2}\right)$ using WinROOF software (version 2.34, Mitani Corp.). The number of extraretinal vascular tufts and the vessel area density in the superficial retina in the OIR model were calculated in thin $z$-section confocal images $\left(5.3 \times 10^{4} \mu \mathrm{m}^{2}\right)$ of 4 random areas, and then averaged per retina. For each group, 4 retinas from 4 mice were analyzed. The unvascularized retinal area in the OIR model was calculated in a panoramic view (Supplemental Figures 7 and 8) using WinROOF software. For each group, 4 retinas from 4 mice were analyzed.

Microarray. Retinal cells dissociated from P8 Tie2GFP Tg mice (47) (The Jackson Laboratory) were separated into GFP-positive ECs and GFP-negative non-ECs by FACSAria (BD). Using total RNA extracted by RNeasy Micro Kit (QIAGEN) and amplified by MessageAmpII aRNA Amplification Kit (Ambion), cRNA was prepared and hybridized onto the Affymetrix oligonucleotide arrays MGU74v2 as described previously (48). Primary data have been deposited in NCBI's Gene Expression Omnibus and are accessible through GEO series accession number GSE27238. The bioinformatics analyses were performed with the eXintegrator system (49) (http://www.cdb.riken.jp/scb/documentation/). Briefly, probe sets representing small GTPase genes were sorted by their similarity (Euclidean distance) to a specified profile with low or nonexistent signals in non-EC samples and high signals in EC samples. The expression data for the resulting list of genes were then manually inspected to remove genes with expression in the non-EC samples and probe sets with ambiguous identities.

Cell culture and transfection. HUVECs (Lonza) cultured in Endothelial Growth Medium EGM-2 BulletKit (Lonza) at a density of $5 \times 10^{3} \mathrm{cells} / \mathrm{cm}^{2}$ in fibronectin-coated culture slides (BD) were transfected with pCAGGSEGFP vector together with PCAGGS vector expressing human RhoJ-WT, RhoJ-CA (G30V) (25), or RhoJ-DN (T35N) (25) tagged with c-Myc at the N-terminus, using TransIT-LT1 (Mirus). After 24 hours, cells were fixed with $4 \% \mathrm{PFA} / \mathrm{PBS}$, incubated with $1 \% \mathrm{BSA} / 0.1 \%$ Triton-X/PBS, and then stained with anti-GFP Ab (Invitrogen), mouse anti-Myc mAb (clone 9E10; Santa Cruz Biotechnology Inc.), and Alexa Fluor 633-phalloidin (Invitrogen). The specimens were observed with an LSM510 confocal microscope (Zeiss). More information on cell culture experiments is provided in Supplemental Methods.

RNA interference. Two predesigned duplexes of Stealth Select RNAi siRNAs (HSS126217 and HSS183814, Invitrogen) targeting different sequences of human RhoJ were transfected to cultured HUVECs using Lipofectamine RNAiMAX according to the manufacture's protocol. Nontargeting siRNA (Stealth RNAi Negative Control Medium GC Duplex, Invitrogen) was used as a negative control. After incubation for 2 days, cells were serum starved with Endothelial Basal Medium EBM-2 (Lonza) containing 0.5\% BSA for 12 hours and then stimulated with human Sema3E $(500 \mathrm{ng} / \mathrm{ml}$, R\&D Systems). After 30 minutes, cells were fixed and stained with Alexa Fluor 488-phalloidin, mouse anti-RhoJ mAb (clone 1E4, Abnova), and TO-PRO-3. Representative data for HSS126217 are presented.

Measurement of RhoJ activity. RhoJ-GTP levels were measured using the colorimetric G-LISA Cdc42 activation assay (Cytoskeleton Inc.). Briefly, HUVECs cultured at a density of $5 \times 10^{3} \mathrm{cells} / \mathrm{cm}^{2}$ in gelatin-coated 60-mm tissue culture dishes (BD) were serum starved for 12 hours, treated with recombinant human VEGF (HumanZyme) and/or human Sema3E (R\&D Systems) for 2 minutes, and lysed according to the manufacturer's protocol. The total protein concentration for each lysate was adjusted to $0.25 \mathrm{mg} / \mathrm{ml}$ using binding buffer and incubated in 96-well plates containing the CRIB domain of the human PAK protein, which can bind to RhoJ-GTP (24), linked to the bottom of each well. After sequential incubation with anti-RhoJ mAb (Abnova), HRP-conjugated secondary Ab, and the HRP detection reagent, the amount of RhoJ-GTP was detected by measuring absorbance at $490 \mathrm{~nm}$. The results are expressed relative to the control (cell lysates without VEGF or Sema3E stimulation) and represent an average of 3 experiments.

Statistics. Statistical analysis was performed with SPSS software (version 10.0J, SPSS Japan Inc.) using 2-tailed, unpaired $t$ tests. $P$ values of less than 0.05 were considered statistically significant.

\section{Acknowledgments}

We thank J. Rossant, T.M. Jessell, C.E. Henderson, and R. Wang for mutant mice; L.M. Jakt and S. Kusuhara for microarray analyses; and S. Koyama for morphometric analyses. We are grateful to the Laboratory for Animal Resources and Genetic Engineering at RIKEN CDB for producing R26-Rhoj chimeric mice and housing the mutant mice. This research was funded by the Global Center of Excellence for Education and Research on Signal Transduction Medicine in the Coming Generation and Grants-in-Aid for Young Scientists (no. 22689046) from the Ministry of Education, Culture, Sports, Science, and Technology (MEXT), Japan. A. Uemura is supported by the Takeda Science Foundation, Mitsubishi Pharma Research Foundation, and the Uehara Memorial Foundation. Y. Yoshida is supported by NIH grant R01NS065048.

Received for publication August 26, 2010, and accepted in revised form February 23, 2011.

Address correspondence to: Akiyoshi Uemura, Division of Vascular Biology, Department of Physiology and Cell Biology, Kobe University Graduate School of Medicine, 7-5-1 Kusunoki-cho, Chuo-ku, Kobe 650-0017, Japan. Phone: 81.78.382.6916; Fax: 81.78.382.5834; E-mail: auemura@med.kobe-u.ac.jp.
1. Friedlander M. Fibrosis and diseases of the eye. J Clin Invest. 2007;117(3):576-586.

2. Gariano RF, Gardner TW. Retinal angiogenesis in development and disease. Nature. 2005; 438(7070):960-966.
3. Saint-Geniez M, D'Amore PA. Development and pathology of the hyaloid, choroidal and retinal vasculature. Int J Dev Biol. 2004;48(8-9):1045-1058.

4. Patz A. Current concepts in ophthalmology. Retinal vascular diseases. N Engl J Med. 1978;
298(26):1451-1454

5. Nicholson BP, Schachat AP. A review of clinical trials of anti-VEGF agents for diabetic retinopathy. Graefes Arch Clin Exp Ophthalmol. 2010;248(7):915-930.

6. Micieli JA, Surkont M, Smith AF. A systematic 
analysis of the off-label use of bevacizumab for severe retinopathy of prematurity. Am J Ophthalmol. 2009;148(4):536-543.

7. Fruttiger M. Development of the retinal vasculature. Angiogenesis. 2007;10(2):77-88.

8. Uemura A, Kusuhara S, Katsuta H, Nishikawa S. Angiogenesis in the mouse retina: a model system for experimental manipulation. Exp Cell Res. 2006; 312(5):676-683.

9. Gerhardt $\mathrm{H}$, et al. VEGF guides angiogenic sprouting utilizing endothelial tip cell filopodia. $J$ Cell Biol. 2003;161(6):1163-1177.

10. Ruhrberg C, et al. Spatially restricted patterning cues provided by heparin-binding VEGF-A control blood vessel branching morphogenesis. Genes Dev. 2002;16(20):2684-2698.

11. Adams RH, Eichmann A. Axon guidance molecules in vascular patterning. Cold Spring Harb Perspect Biol. 2010;2(5):a001875.

12. Chauvet S, et al. Gating of Sema3E/PlexinD1 signaling by neuropilin-1 switches axonal repulsion to attraction during brain development. Neuron. 2007;56(5):807-822.

13. Bellon A, et al. VEGFR2 (KDR/Flk1) signaling mediates axon growth in response to semaphorin $3 \mathrm{E}$ in the developing brain. Neuron. 2010;66(2):205-219.

14. Gu C, et al. Semaphorin $3 \mathrm{E}$ and plexin-D1 control vascular pattern independently of neuropilins. Science. 2005;307(5707):265-268.

15. Uesugi K, Oinuma I, Katoh H, Negishi M. Different requirement for Rnd GTPases of R-Ras GAP activity of Plexin-C1 and Plexin-D1. J Biol Chem. 2009; 284(11):6743-6751.

16. Sakurai A, et al. Semaphorin $3 \mathrm{E}$ initiates antiangiogenic signaling through plexin D1 by regulating Arf6 and R-Ras. Mol Cell Biol. 2010;30(12):3086-3098.

17. Stone J, et al. Development of retinal vasculature is mediated by hypoxia-induced vascular endothelial growth factor (VEGF) expression by neuroglia. J Neurosci. 1995;15(7 pt 1):4738-4747.

18. West H, Richardson WD, Fruttiger M. Stabilization of the retinal vascular network by reciprocal feedback between blood vessels and astrocytes. Development. 2005;132(8):1855-1862.

19. Shibuya M. Vascular endothelial growth factor receptor-1 (VEGFR-1/Flt-1): a dual regulator for angiogenesis. Angiogenesis. 2006;9(4):225-230.

20. Moriya J, et al. Inhibition of semaphorin as a novel strategy for therapeutic angiogenesis. Circ Res. 2010; 106(2):391-398.

21. Kruger RP, Aurandt J, Guan KL. Semaphorins command cells to move. Nat Rev Mol Cell Biol. 2005;
6(10):789-800.

22. Hall A, Lalli G. Rho and Ras GTPases in axon growth, guidance, and branching. Cold Spring Harb Perspect Biol. 2010;2(2):a001818.

23. Puschel AW. GTPases in semaphorin signaling. $A d v$ Exp Med Biol. 2007;600:12-23.

24. Vignal E, et al. Characterization of TCL, a new GTPase of the rho family related to TC10 and Cdc42.J Biol Chem. 2000;275(46):36457-36464.

25. Abe T, Kato M, Miki H, Takenawa T, Endo T. Small GTPase Tc10 and its homologue RhoT induce NWASP-mediated long process formation and neurite outgrowth. J Cell Sci. 2003;116(pt 1):155-168.

26. Bustelo XR, Sauzeau V, Berenjeno IM. GTP-binding proteins of the Rho/Rac family: regulation, effectors and functions in vivo. Bioessays. 2007; 29(4):356-370.

27. Heasman SJ, Ridley AJ. Mammalian Rho GTPases: new insights into their functions from in vivo studies. Nat Rev Mol Cell Biol. 2008;9(9):690-701.

28. Niwa H, Yamamura K, Miyazaki J. Efficient selection for high-expression transfectants with a novel eukaryotic vector. Gene. 1991;108(2):193-199.

29. Xiao C, et al. Lymphoproliferative disease and autoimmunity in mice with increased miR-17-92 expression in lymphocytes. Nat Immunol. 2008; 9(4):405-414.

30. Braren R, Hu H, Kim YH, Beggs HE, Reichardt LF, Wang R. Endothelial FAK is essential for vascular network stability, cell survival, and lamellipodial formation. J Cell Biol. 2006;172(1):151-162.

31. Egawa G, Osawa M, Uemura A, Miyachi Y, Nishikawa $\mathrm{S}$. Transient expression of ephrin B2 in perinatal skin is required for maintenance of keratinocyte homeostasis. J Invest Dermatol. 2009;129(10):2386-2395.

32. Smith LE, et al. Oxygen-induced retinopathy in the mouse. Invest Ophthalmol Vis Sci. 1994;35(1):101-111.

33. Albuquerque RJ, et al. Alternatively spliced vascular endothelial growth factor receptor- 2 is an essential endogenous inhibitor of lymphatic vessel growth. Nat Med. 2009;15(9):1023-1030.

34. Shalaby F, et al. Failure of blood-island formation and vasculogenesis in Flk-1-deficient mice. Nature. 1995;376(6535):62-66.

35. Zhang Y, et al. Tie2Cre-mediated inactivation of plexinD1 results in congenital heart, vascular and skeletal defects. Dev Biol. 2009;325(1):82-93.

36. Ferrara N, Gerber HP, LeCouter J. The biology of VEGF and its receptors. Nat Med. 2003;9(6):669-676.

37. Lamalice L, Houle F, Jourdan G, Huot J. Phosphorylation of tyrosine 1214 on VEGFR2 is required for VEGF-induced activation of Cdc42 upstream of
SAPK2/p38. Oncogene. 2004;23(2):434-445.

38. Gitler AD, Lu MM, Epstein JA. PlexinD1 and semaphorin signaling are required in endothelial cells for cardiovascular development. Dev Cell. 2004; 7(1):107-116

39. Aiello LP, et al. Vascular endothelial growth factor in ocular fluid of patients with diabetic retinopathy and other retinal disorders. N Engl J Med. 1994; 331(22):1480-1487.

40. Sennlaub F, Courtois Y, Goureau O. Inducible nitric oxide synthase mediates the change from retinal to vitreal neovascularization in ischemic retinopathy. J Clin Invest. 2001;107(6):717-725.

41. Skoura A, Sanchez T, Claffey K, Mandala SM, Proia RL, Hla T. Essential role of sphingosine 1-phosphate receptor 2 in pathological angiogenesis of the mouse retina. J Clin Invest. 2007; 117(9):2506-2516

42. FitzGibbon T. The human fetal retinal nerve fiber layer and optic nerve head: a DiI and DiA tracing study. Vis Neurosci. 1997;14(3):433-447.

43. Dorrell MI, et al. Maintaining retinal astrocytes normalizes revascularization and prevents vascular pathology associated with oxygen-induced retinopathy. Glia. 2010;58(1):43-54.

44. Roodink I, Verrijp K, Raats J, Leenders WP. Plexin D1 is ubiquitously expressed on tumor vessels and tumor cells in solid malignancies. BMC Cancer. 2009;9:297.

45. Uemura A, Kusuhara S, Wiegand SJ, Yu RT, Nishikawa S. Tlx acts as a proangiogenic switch by regulating extracellular assembly of fibronectin matrices in retinal astrocytes. J Clin Invest. 2006; 116(2):369-377.

46. Watakabe A, Ohsawa S, Hashikawa T, Yamamori $\mathrm{T}$. Binding and complementary expression patterns of semaphorin $3 \mathrm{E}$ and plexin D1 in the mature neocortices of mice and monkeys. J Comp Neurol. 2006;499(2):258-273.

47. Motoike T, et al. Universal GFP reporter for the study of vascular development. Genesis. 2000; 28(2):75-81.

48. Takebe A, Era T, Okada M, Jakt LM, Kuroda Y, Nishikawa S. Microarray analysis of PDGFR alpha+ populations in ES cell differentiation culture identifies genes involved in differentiation of mesoderm and mesenchyme including ARID3b that is essential for development of embryonic mesenchymal cells. Dev Biol. 2006;293(1):25-37.

49. Jakt LM, Nishikawa S. DNA chip databases, omics, and gene fishing: commentary. Cancer Sci. 2008;99(5):829-835. 